\title{
INVARIANT THEOREMS IN EUCLIDEAN GEOMETRY WITH RESPECT TO CONICS
}

\author{
Veselin Nenkov
}

\begin{abstract}
Research is usually preceded by natural aspiration to discover new knowledge based on well-known facts. Many scientific facts are known for centuries, however new peculiarities are discovered by contemporary means. Information technologies together with abundant arsenal of knowledge and skills for effective application initiate specific way of thinking. The dynamic geometric software turns out to be a basic instrument to study objects from the Euclidean geometry. The present paper uses the possibilities of "THE GEOMETER'S SKETCHPAD” (GSP) in the generalization of some classic and some not very popular theorems after analyzing basic properties of the objects under study.
\end{abstract}

\section{INTRODUCTION}

In the plane of a given triangle $A B C$ some special points are determined, which characterize various properties of the triangle. Such points are the orthocentre, the in-centre, the circum-centre and ex-centres. Remarkable relations, which are connected with these six points (in the general case), are the Euler line, the Euler circle of the $\triangle A B C$ and the isogonal conjugates with respect to $\triangle A B C$. It is curious to examine whether in substituting any of the mentioned points by arbitrary one from the plane of the $\triangle A B C$ (with possible exceptions of natural character), the other five points are determined uniquely, thus transforming the known lines and circles into generalizations with similar properties. We propose an analysis of some characteristic properties of the already mentioned notable points of the triangle by an essential assistance of the GSP, and we show the existence of corresponding generalizations, which are analogous to already known figures from the geometry of the triangle.

2010 Mathematics Subject Classification. Primary: 51-04, 68T01, 68T99.

Key words and phrases. Feuerbach Point, Euler curve 
In such a way we demonstrate the joint work of the analogy and the computer software GSP to notice generalizations of well-known theorems from the geometry of the triangle. By the help of the GSP we examine the dependence of some triangle properties on an arbitrary point. The whole process should be considered as finalized only in case the results are proved in a strictly mathematical way. The proofs are elaborated using barycentric coordinates with respect to the given triangle $A B C$, namely $A(1,0,0), B(0,1,0)$ and $C(0,0,1)$

[1]. The midpoints of the sides $B C, C A$ and $A B$ are denoted by $M_{a}\left(0, \frac{1}{2}, \frac{1}{2}\right)$, $M_{b}\left(\frac{1}{2}, 0, \frac{1}{2}\right)$ and $M_{c}\left(\frac{1}{2}, \frac{1}{2}, 0\right)$, respectively.

\section{EULER LINE AND EULER CURVE, DEPENDING ON A POINT}

Every non-equilateral triangle has a special line, known to be Euler line and also a special circle - Euler circle [1], [2]. Following reasoning by analogy we will show by means of the computer program GSP how a similar line and a curve of second degree for a given triangle could be obtained in dependence on an arbitrary point in the plane of the triangle.

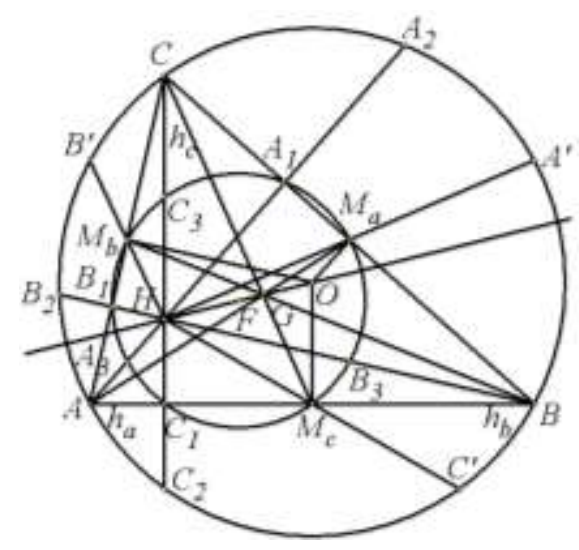

Figure 1

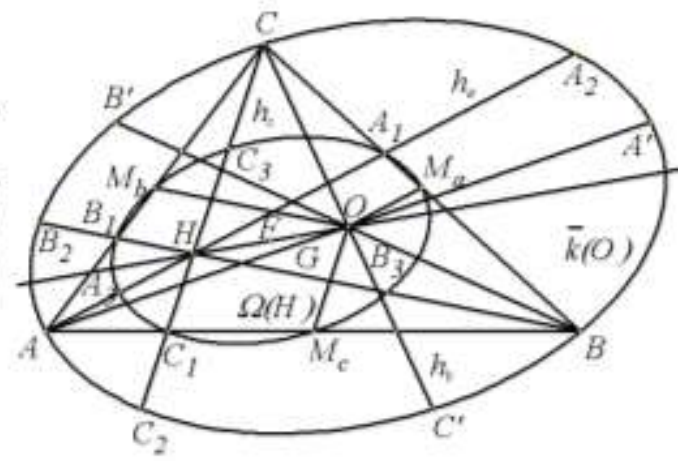

Figure 2

In the general case, characteristic points of $\triangle A B C$ on the Euler line are the circum-centre, the orthocentre, the gravity centre and the centre of the Euler circle (Fig. 1). The circum-circle of the $\triangle A B C$ is one element only of an infinity set of second degree curves, which are circumscribed round $\triangle A B C$. Something more, if $O\left(x_{0}, y_{0}, z_{0}\right)\left(x_{0}+y_{0}+z_{0}=1\right)$ is an arbitrary point, which 
is not concurrent with the lines $B C, C A, A B, M_{b} M_{c}, M_{c} M_{a}$ and $M_{a} M_{b}$ in the plane of the $\triangle A B C$, then $A, B, C$ and correspondingly their symmetric points with respect to $O$

$$
A^{\prime}\left(2 x_{0}-1,2 y_{0}, 2 z_{0}\right), B^{\prime}\left(2 x_{0}, 2 y_{0}-1,2 z_{0}\right), C^{\prime}\left(2 x_{0}, 2 y_{0}, 2 z_{0}-1\right)
$$

lie on a curve of second degree $\bar{k}(O)$ with centre $O$ (Fig. 2, 3). The curve $\bar{k}(O)$ has the following equation:

$$
\bar{k}(O):\left(1-2 x_{0}\right) x_{0} y z+\left(1-2 y_{0}\right) y_{0} z x+\left(1-2 z_{0}\right) z_{0} x y=0 .
$$

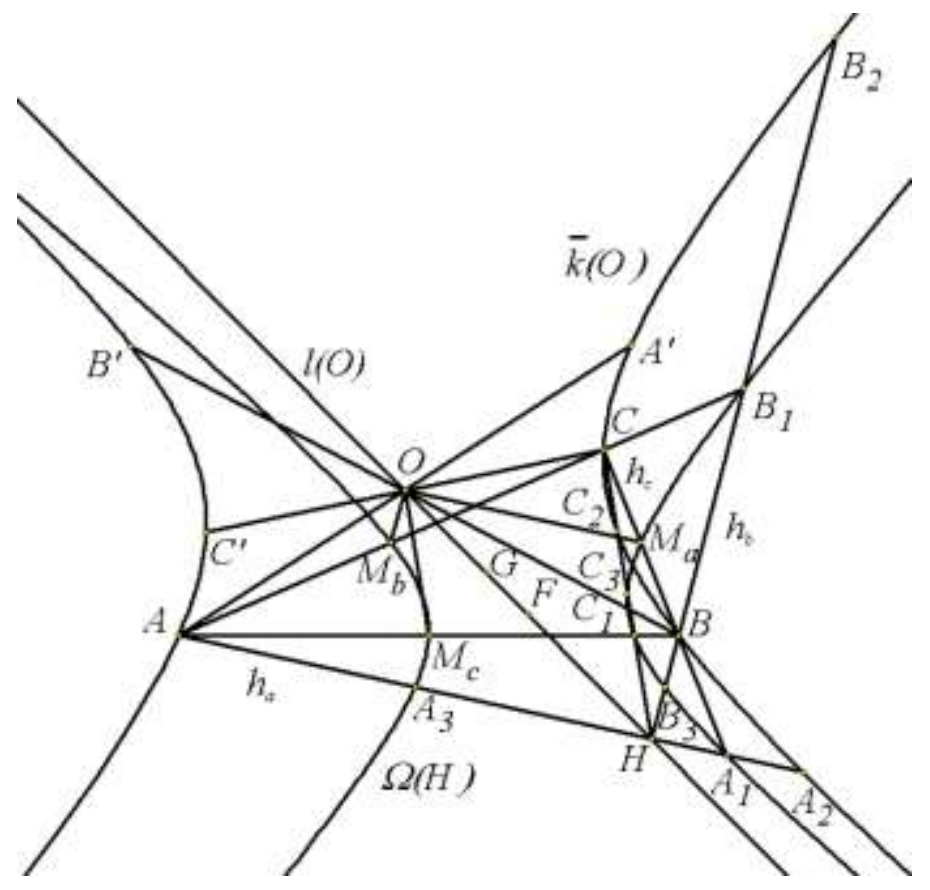

Figure 3

The point $O$, thus determined is analogous to the circum-centre of $\triangle A B C$. Now, we will define a point, which is analogous to the orthocentre of $\triangle A B C$. The altitudes of $\triangle A B C$ are parallel to the lines, which pass through the circumcentre and the points $M_{a}, M_{b}$ and $M_{c}$ (Fig. 1). This is a reason to construct the lines $h_{a}, h_{b}$ and $h_{c}$ by the GSP, passing through the vertices $A, B, C$, respectively and parallel to the lines $O M_{a}, O M_{b}$ and $O M_{c}$, respectively. It is seen that these lines have a common point $H$ (Fig. 2, 3). Additionally, no matter how the position of $O$ is changed, the lines under consideration have a common point always. In such a way the following assertion is obtained: 
Property 1. The lines $h_{a}, h_{b}$ and $h_{c}$ have a common point $H$.

For the proof we find parametric equations of the lines $h_{a}, h_{b}$ and $h_{c}$ :

$$
\begin{aligned}
& h_{a}: x=1+x_{0} t_{a}, y=\left(y_{0}-\frac{1}{2}\right) t_{a}, z=\left(z_{0}-\frac{1}{2}\right) t_{a}, \\
& h_{b}: x=\left(x_{0}-\frac{1}{2}\right) t_{b}, y=1+y_{0} t_{b}, z=\left(z_{0}-\frac{1}{2}\right) t_{b}, \\
& h_{c}: x=\left(x_{0}-\frac{1}{2}\right) t_{c}, y=\left(y_{0}-\frac{1}{2}\right) t_{c}, z=1+z_{0} t_{c} .
\end{aligned}
$$

Further, we solve the system with the equations of the lines $h_{a}$ and $h_{b}$. We find that the common point is $H\left(1-2 x_{0}, 1-2 y_{0}, 1-2 z_{0}\right)$. Finally, we check that the coordinates of this point satisfy the equations of $h_{c}$.

For a more convincing analogy between the point $H$ and the orthocentre of $\triangle A B C$, this point should possess other properties, which are characteristic for the orthocentre. Let the lines $h_{a}, h_{b}$ and $h_{c}$ intersect the lines $B C, C A$ and $A B$ in the points $A_{1}, B_{1}$ and $C_{1}$, respectively, and also the curve $\bar{k}(O)-$ in the points $A_{2}, B_{2}$ and $C_{2}$, respectively (Fig. 2, 3). It is well-known that the points, which are symmetric to the orthocentre with respect to the sides and the midpoints of the sides, lie of the circum-circle of $\triangle A B C$ (Fig. 1) [1]. For this reason we check by the GSP whether the points $A_{2}, B_{2}$ and $C_{2}$ are symmetric to $H$ with respect to $A_{1}, B_{1}$ and $C_{1}$, correspondingly. We check also whether the points $A^{\prime}, B^{\prime}$ and $C^{\prime}$ are symmetric to $H$ with respect to $M_{a}, M_{b}$ and $M_{c}$, correspondingly. All constructions lead to the conclusion that $H$ has the following properties:

Property 2. The points $A_{2}, B_{2}$ and $C_{2}$ are symmetric to $H$ with respect to $A_{1}, B_{1}$ and $C_{1}$, correspondingly.

Property 3. The points $A^{\prime}, B^{\prime}$ and $C^{\prime}$ are symmetric to $H$ with respect to $M_{a}, M_{b}$ and $M_{c}$, correspondingly.

The proofs of these properties could be obtained using the coordinates of the coresponding ponts.

Because of the shown analogy of the constructed point $H$ with the orthocentre of $\triangle A B C$, we will call this point to be orthoid of $\triangle A B C$, depending on the point $O$. 
In the GSP let us construct now the line $O H$ and the gravity centre $G\left(\frac{1}{3}, \frac{1}{3}, \frac{1}{3}\right)$ of $\triangle A B C$. We establish, that:

Property 4. The points $O, H$ and $G$ are collinear and the point $G$ divides the segment $\mathrm{HO}$ in ratio $2: 1$.

The proof ot this property could be obtained by a reasoning, that the coordinates of the points $O, H$ and $G$ imply the vector equality $\overrightarrow{H G}=2 \cdot \overrightarrow{G O}$. The property 4 shows, that the line $l(O)$, containing the points $O, H$ and $G$ is an analogue to the Euler line and for this reason we will say, that $l(O)$ is Euler line of $\triangle A B C$, depending on the point $O$.

The centre of the Euler circle of $\triangle A B C$ lies on the Euler line too. Let us look for a curve, which is analogous to the Euler circle. In the general case the Euler circle contains the points $M_{a}, M_{b}$ and $M_{c}$, the feet of the altitudes and the midpoints of the segments connecting the vertices of the triangle with its orthocentre (Fig. 1) [1].

For an arbitrary point $P(\lambda, \mu, v)(\lambda+\mu+v=1)$ from the plane of $\triangle A B C$, the points $M_{a}, M_{b}$ and $M_{c}$, the common points of the lines $A P, B P$ and $C P$, denoted with $A_{3}, B_{3}$ and $C_{3}$, respectively, lie on a curve of second degree $\Omega(P)$ (Fig. 2, 3). We call it to be Euler curve for the point $P$ with respect to $\triangle A B C$. This curve has the following equation:

$\Omega(P): \mu v x^{2}+v \lambda y^{2}+\lambda \mu z^{2}-(1-\lambda) \lambda y z-(1-\mu) \mu z x-(1-v) v x y=0$.

Obviously, the Euler circle of $\triangle A B C$ is Euler curve of its orthocentre. Since $H$ is an analogue to the orthocentre, it is reasonable in this case to consider the Euler curve $\Omega(H)$ of the point $H$. It follows from the definition, that $\Omega(H)$ passes through the points $M_{a}, M_{b}, M_{c}, A_{1}, B_{1}, C_{1}$ and the midpoints of the segments $A H, B H$ and $C H$, and its equation is

$$
\begin{aligned}
& \left(1-2 y_{0}\right)\left(1-2 z_{0}\right) x^{2}+\left(1-2 z_{0}\right)\left(1-2 x_{0}\right) y^{2}+\left(1-2 x_{0}\right)\left(1-2 y_{0}\right) z^{2}- \\
& -2\left(1-2 x_{0}\right) x_{0} y z-2\left(1-2 y_{0}\right) y_{0} z x-2\left(1-2 z_{0}\right) z_{0} x y=0 .
\end{aligned}
$$

We say, that $\Omega(H)$ is Euler curve of $\triangle A B C$, depending on the point $O$. Experimenting by the GSP we establish for the points $A_{3}, B_{3}$ and $C_{3}$, that

Property 5. The lines $A^{\prime} A_{3}, B^{\prime} B_{3}$ and $C^{\prime} C_{3}$, pass through the point $G$ and the relations $G A^{\prime}: G A_{3}=G B^{\prime}: G B_{3}=G C^{\prime}: G C_{3}=2: 1$ are satisfied. 
The coordinates of the points under consideration imply the vector equalities $\overrightarrow{G A_{3}}=-\frac{1}{2} \overrightarrow{G A^{\prime}}, \overrightarrow{G B_{3}}=-\frac{1}{2} \overrightarrow{G B^{\prime}}$ and $\overrightarrow{G C_{3}}=-\frac{1}{2} \overrightarrow{G C^{\prime}}$, which prove the property.

Now, let $h\left(H, \frac{1}{2}\right)$ be a homothety with centre $H$ and coefficient $\frac{1}{2}$, while $h\left(G,-\frac{1}{2}\right)$ be a homothety with centre $O$ and $-\frac{1}{2}$. We obtain from properties 2 , 3,5 and the main property of the gravity centre, that:

Property 6. The homotheties $h\left(H, \frac{1}{2}\right)$ and $h\left(G,-\frac{1}{2}\right)$ transform $\bar{k}(O)$ to $\Omega(H)$.

It follows directly from the last property, that

Property 7. The curves $\bar{k}(O)$ and $\Omega(H)$ are of the same type.

Let $F\left(\frac{1-x_{0}}{2}, \frac{1-y_{0}}{2}, \frac{1-z_{0}}{2}\right)$ be the midpoint of the segment $O H$. It follows from property 6 , that

Property 8. The point $F$ is centre of the Euler curve $\Omega(H)$.

We have also the following

Property 9. The homotheties $h\left(H, \frac{1}{2}\right)$ and $h\left(G,-\frac{1}{2}\right)$ transform all points from $\bar{k}(O)$ to diametrically opposite points from $\Omega(H)$.

The obtained results imply, that the line $l(O)$ does not exist exactly when $O \equiv G$. In this case $\Omega(G)$ is an ellipse, inscribed in $\triangle A B C$. This fact explains why the equilateral triangle has no Euler line.

Up to now, we have considered the cases, when the circum-curve of $\triangle A B C$ is an ellipse or a hyperbola with center in a given point $O$. The circum parabolas of $\triangle A B C$ could be considered as conic sections with infinity centers [3]. The infinity center $O$ of the parabola could be determined by the directrix of a given vector $\vec{O}$. Let $\vec{O}\left(x_{0}, y_{0}, z_{0}\right)\left(x_{0}+y_{0}+z_{0}=0\right)$ be a vector, which is 
not collinear with any of the lines $B C, C A$ and $A B$. A unique parabola $k(\vec{O})$ exists, which passes through the points $A, B$ and $C$. It has an axis, which is a collinear line with $\vec{O}$ (it touches the infinity line of the plane in the infinity point $\vec{O})$ [3]. The parabola $k(\vec{O})$ has the following equation:

$$
k(\vec{O}): x_{0}^{2} y z+y_{0}^{2} z x+z_{0}^{2} x y=0 .
$$

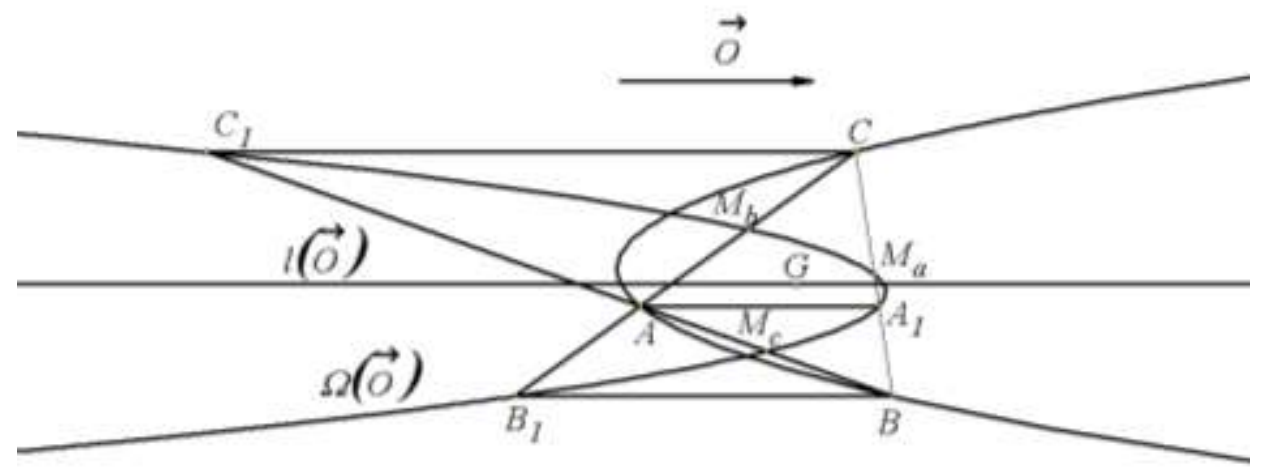

Figure 4

In this case the point $H$ could be considered as coinciding with the infinity center of $k(\vec{O})$. We consider the line $l(\vec{O})$ through $G$ and collinear with $\vec{O}$ to be Euler line of $\triangle A B C$, depending on the directrix $\vec{O}$ (or, which is the same, depending on the infinity point $\vec{O}$, denoting it by $l(\vec{O}))$.

We construct the lines $a_{0}, b_{0}$ and $c_{0}$, which pass through the vertices $A$, $B$ and $C$, correspondingly and are collinear with the vector $\vec{O}$. Let $a_{0} \cap B C=A_{1}, b_{0} \cap C A=B_{1}$ and $c_{0} \cap A B=C_{1}$. The coordinates of the points $M_{a}, M_{b}, M_{c}, A_{1}, B_{1}, C_{1}$ and the vector $\vec{O}$ satisfy the equation

$$
\Omega(\vec{O}): y_{0} z_{0} x^{2}+z_{0} x_{0} y^{2}+x_{0} y_{0} z^{2}+x_{0}^{2} y z+y_{0}^{2} z x+z_{0}^{2} x y=0 .
$$

The parabola $\Omega(\vec{O})$ is considered to be Euler curve of $\triangle A B C$, depending on the direcrix $\vec{O}$ (or, which is the same, depending on the infinity point).

The parabolas $k(\vec{O})$ and $\Omega(\vec{O})$ are connected with the following

Property 10. The homothety $h\left(G,-\frac{1}{2}\right)$ transforms the parabola $\Omega(\vec{O})$ into the parabola $k(\vec{O})$. 
The last property shows, that the circum parabolas of $\triangle A B C$ look like the circum ellipse of $\triangle A B C$ with center in the point $G$ because in each of the two cases there is only one homothety, transforming the circum curve into the corresponding Euler curve.

Notice, that the infinity points also generate Euler lines and Euler curves (parabolas) of the given triangle. However, in this case the properties are less abundant because they are connected with circum parabolas round the triangle. The parabolas are placed more especially in the set of the three conic sections with respect to the centre notion. For this reason they transfer the peculiarity to the questions under consideration.

The above investigations show, that Euler line $l(O)$ and Euler curve $\Omega(H)$ could be associated to each finite or infinite point $O$ from the plane of a given triangle $A B C$, which is not concurrent with any of the lines $B C, C A, A B$, $M_{b} M_{c}, M_{c} M_{a}, M_{a} M_{b}$ and which is different from the gravity center $G$. The line $l(O)$ and the curve $\Omega(H)$ coincide with the classic ones exactly when $O$ is the circumcenter of a non-equilateral triangle $\triangle A B C$.

\section{GENERALIZATION OF THE FEUERBACH THEOREM}

A well-known Feuerbach theorem from the geometry of the triangle asserts, that the Euler circle of a triangle is tangent to the four in-circles of this triangle [2], [4]. What follows in the sequel is a generalization of this theorem.

By analogous reasoning, notifying some main dependences among the circles from the Feuerbach theorem, we will show how to obtain a generalization of this theorem by the computer program GSP, in which the participation of circles is not obligatory. Firstly, we will notify the generation of a triangle, which is conjugated to arbitrary point $P$ with respect to the given $\triangle A B C$.

Let the points $A_{1}, B_{1}$ and $C_{1}$ be on the lines $B C, C A$ and $A B$, respectively and be such, that the lines $A A_{1}, B B_{1}$ and $C C_{1}$, pass through a point $P$ (finite or infinite). If the points $A_{2}, B_{2}$ and $C_{2}$ are the harmonic conjugated to $A_{1}, B_{1}$ and $C_{1}$, respectively, with respect to the couples of vertices of the given triangle, then the lines $A A_{2}, B B_{2}$ and $C C_{2}$ are called to be harmonic conjugated to the lines $A P, B P$ and $C P$ with respect to $\triangle A B C$ [3]. Let $B B_{2} \cap C C_{2}=P_{a}, \quad C C_{2} \cap A A_{2}=P_{b}$ and $A A_{2} \cap B B_{2}=P_{c}$, then the triangle $\triangle P_{a} P_{b} P_{c}$ is called to be conjugated (harmonic) to the point $P$ with respect to 
$\triangle A B C$ [1]. It is easy to show, that the conjugated triangle of $P_{a}$ is $\Delta P_{b} P_{c} P$, of $P_{b}$ is $\Delta P_{c} P_{a} P$, and of $P_{c}$ is $\Delta P_{a} P_{b} P$ [1]. For this reason we will say for such four points in the plane of $\triangle A B C$, that they form a harmonic four of points with respect to $\triangle A B C$.

The first thing, on which we should pay attention in the Feuerbach theorem, is that five circles participate in it and four of them are tangent to the lines $B C$, $C A$ and $A B$. Since the circle is a special type ellipse and the ellipse is a type central conic section, then the desired generalization should be in search through the inscribed conic sections of the given triangle. At the beginning the generalization will be in searching through ellipses and hyperbolas, after that through the inscribed parabolas.

The next thing, on which we should pay attention, is that the four in-circles participate in the Feuerbach theorem jointly and not separately - each for itself. Consequently, their centers participate jointly too. A remarkable property of the four centers is that they form a harmonic four of points with respect to $\triangle A B C$.

Construct a triangle $A B C$ in the GSP program and also an arbitrary harmonic four $I, I_{A}, I_{B}$ and $I_{C}$. Let $I\left(x_{I}, y_{I}, z_{I}\right), I_{A}\left(-\frac{x_{I}}{1-2 x_{I}}, \frac{y_{I}}{1-2 x_{I}}, \frac{z_{I}}{1-2 x_{I}}\right)$, $I_{B}\left(\frac{x_{I}}{1-2 x_{I}},-\frac{y_{I}}{1-2 x_{I}}, \frac{z_{I}}{1-2 x_{I}}\right)$ and $I_{C}\left(\frac{x_{I}}{1-2 x_{I}}, \frac{y_{I}}{1-2 x_{I}},-\frac{z_{I}}{1-2 x_{I}}\right)$ [1] be points, which form a harmonic four of points with respect to $\triangle A B C$.

Another remarkable property of the in-centers is that they define segments, whose midpoints lie on the circum-circle of $\triangle A B C$. For this reason we consider the midpoints $M_{A}, M_{B}, M_{C}, M_{B C}, M_{C A}$ and $M_{A B}$ of the segments of $I I_{A}$, $I I_{B}, I I_{C}, I_{B} I_{C}, I_{C} I_{A}$ and $I_{A} I_{B}$, respectively. Now, we should expect, that the points $M_{A}, M_{B}, M_{C}, M_{B C}, M_{C A}$ and $M_{A B}$ lie on a central conic section, circumscribed round $\triangle A B C$. The case when the points $I, I_{A}, I_{B}$ and $I_{C}$ are the centers of the in-circles of $\triangle A B C$, the points $M_{A}, M_{B}, M_{C}$, $M_{B C}, M_{C A}$ and $M_{A B}$ are midpoints of arcs on the circum-circle of $\triangle A B C$. The perpendicular bisectors of the segments $B C, C A$ and $A B$ pass through the same midpoints too. On the other hand, these perpendicular bisectors pass also through the midpoints $M_{a}, M_{b}, M_{c}$ of the segments $B C, C A$ and $A B$, respectively. Therefore, we could expect, that in this case the lines $M_{A} M_{a}$, $M_{B} M_{b}$ and $M_{C} M_{c}$ are analogous to the perpendicular bisectors and for this reason they should have a common point. Construct the lines $M_{A} M_{a}, M_{B} M_{b}$ 
and $M_{C} M_{c}$ in the computer program GSP. The construction shows, that these lines pass through a point $O\left(x_{0}, y_{0}, z_{0}\right)\left(x_{0}+y_{0}+z_{0}=1\right)$ and $M_{B C} \in M_{A} M_{a}$, $M_{C A} \in M_{B} M_{b}$ and $M_{A B} \in M_{C} M_{c}$, which visualizes the correctness of the supposition. The analytic computations in [9] confirm the observed concurrence. Additionally, it is established that the points $M_{A}, M_{B}, M_{C}$, $M_{B C}, M_{C A}$ and $M_{A B}$ lie on the circumscribed conic section $\bar{k}(O)$ of $\triangle A B C$ with equation (1), while the coordinates of its center are determined by the equalities

$$
\begin{aligned}
& x_{0}=\frac{\left(1-2 x_{I}-2 y_{I} z_{I}\right) x_{I}^{2}}{\left(1-2 x_{I}\right)\left(1-2 y_{I}\right)\left(1-2 z_{I}\right)}, \\
& y_{0}=\frac{\left(1-2 y_{I}-2 z_{I} x_{I}\right) y_{I}^{2}}{\left(1-2 x_{I}\right)\left(1-2 y_{I}\right)\left(1-2 z_{I}\right)}, \\
& z_{0}=\frac{\left(1-2 z_{I}-2 x_{I} y_{I}\right) z_{I}^{2}}{\left(1-2 x_{I}\right)\left(1-2 y_{I}\right)\left(1-2 z_{I}\right)} .
\end{aligned}
$$

Another property of the in-centers is that the lines determined by the centers and the tangent points are perpendicular to the lines $B C, C A$ and $A B$. For this reason these lines are parallel to the perpendicular bisectors of the segments $B C, C A$ and $A B$. Use the notations $k(I), k\left(I_{A}\right), k\left(I_{B}\right)$ and $k\left(I_{C}\right)$ for the inscribed conic sections of $\triangle A B C$, whose centers are the points $I, I_{A}, I_{B}$ and $I_{C}$, respectively. Further we will examine $k(I)$ only, because the remaining inscribed conic sections have the same properties.

Use the notations $A_{I}, B_{I}$ and $C_{I}$ for the tangent points of $k(I)$ with the lines $B C, C A$ and $A B$, respectively. It follows from the already done observations, that the lines $I A_{I}, I B_{I}$ and $I C_{I}$ should be parallel to the lines $O M_{a}, O M_{b}$ and $O M_{c}$, respectively. Thus, using the GSP program we construct the lines $I A_{I}, I B_{I}$ and $I C_{I}$ through the point $I$, which are parallel to the lines $O M_{a}, O M_{b}$ and $O M_{c}$, respectively. Further, we construct the points $A_{I}, B_{I}$ and $C_{I}$, as intersections of the lines $I A_{I}, I B_{I}$ and $I C_{I}$, with the lines $B C, C A$ and $A B$, respectively and also the curve $k(I)$, which passes through these points and whose center is the point $I$. We see, that $k(I)$ has no other common point with the lines $B C, C A$ and $A B$. Consequently, $k(I)$ is an inscribed curve of $\triangle A B C$. The observations are proved strictly in [5], where it is shown, that the points $A_{I}, B_{I}$ and $C_{I}$ lie on the in-curve $k(I)$ of $\triangle A B C$, 
whose equation is the following:

$$
\begin{aligned}
& \left(1-2 x_{I}\right)^{2} x^{2}+\left(1-2 y_{I}\right)^{2} y^{2}+\left(1-2 z_{I}\right)^{2} z^{2}-2\left(1-2 y_{I}\right)\left(1-2 z_{I}\right) y z- \\
& -2\left(1-2 z_{I}\right)\left(1-2 x_{I}\right) z x-2\left(1-2 x_{I}\right)\left(1-2 y_{I}\right) x y=0 .
\end{aligned}
$$

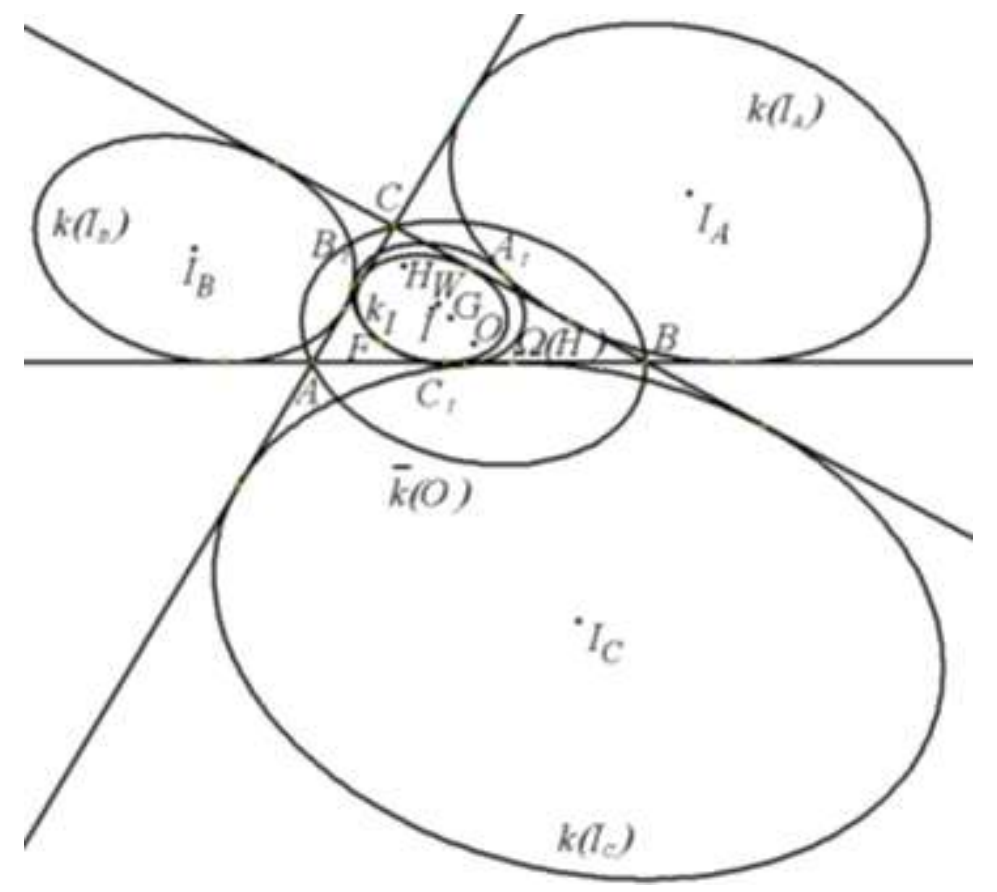

Figure 5

Let $H$ be the point depending on $O$ by which $O$ determines the Euler line $l(O)$. Construct the Euler curve $\Omega(H)$ for the point $H$ using the computer program GSP. Notice, that this curve is tangent to $k(I), k\left(I_{A}\right), k\left(I_{B}\right)$ and $k\left(I_{C}\right)$ consequently, this is the generalization of the Feuerbach theorem in search. Thus, it is confirmed one more time again, that $\Omega(H)$ is the true analogue to the Euler circle, which depends on a point from the plane of $\triangle A B C$ . After using the equations of the curves $k(I), \Omega(H)$ and also the dependence among the coordinates of the centers $O$ and $I$, it is shown in в [5], that $k(I)$ and $\Omega(H)$ are tangent in the point

$$
F\left(\frac{\left(2 x_{I}-1\right)\left(y_{I}-z_{I}\right)^{2}}{f}, \frac{\left(2 y_{I}-1\right)\left(z_{I}-x_{I}\right)^{2}}{f}, \frac{\left(2 z_{I}-1\right)\left(x_{I}-y_{I}\right)^{2}}{f}\right),
$$


where $f=2\left(\left(1-2 x_{I}\right)\left(1-2 y_{I}\right)\left(1-2 z_{I}\right)-x_{I} y_{I} z_{I}\right)$.

In such a way, the following generalization of the Feuerbach theorem is established:

Theorem 1. The inscribed conic sections $k(I), k\left(I_{A}\right), k\left(I_{B}\right)$ and $k\left(I_{C}\right)$ of $\triangle A B C$ are tangent to the Euler curve $\Omega(H)$ (Fig. 5).

It is seen from the constructions, already performed in the computer program GSP, that the five curves are ellipses or hyperbolas simultaneously. Something more, when they are hyperbolas it seems that they have the same asymptotic directions. It could be said in fact, that the five hyperbolas pass through two infinite points. The proof of these facts is performed in [5].

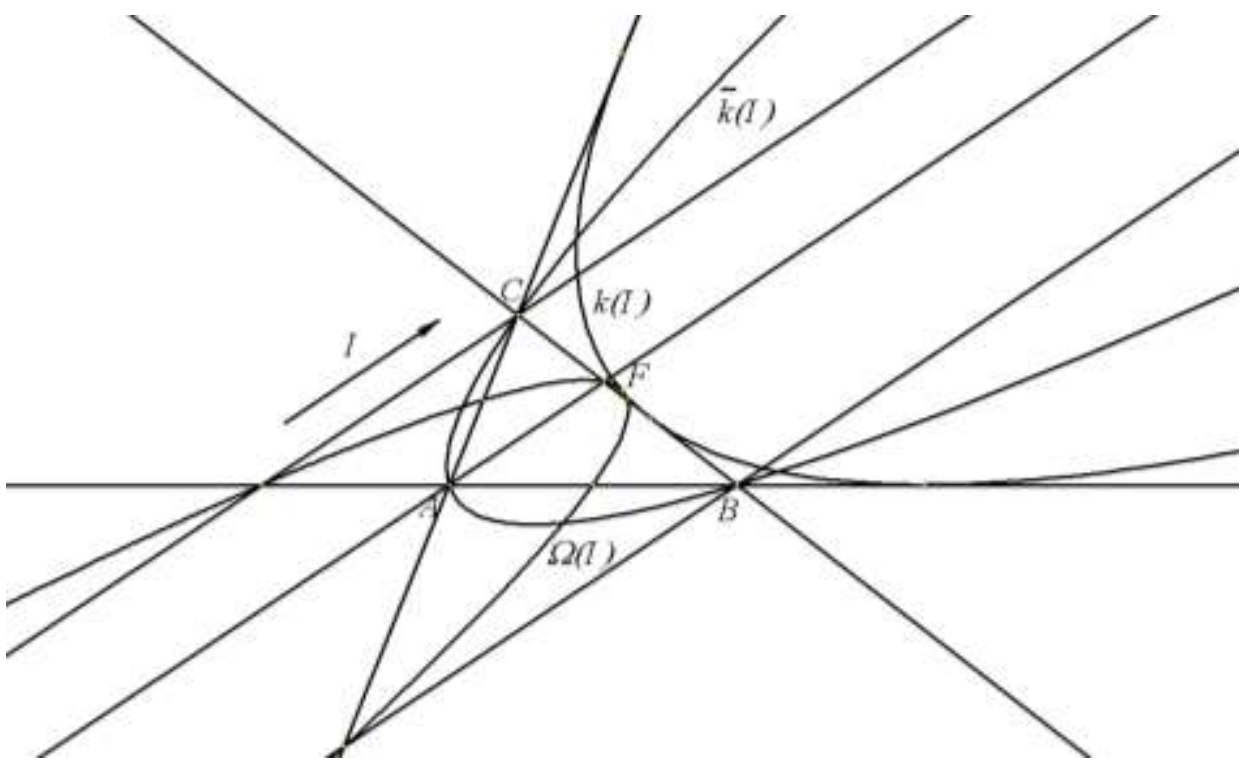

Figure 6

Now let $I\left(x_{I}, y_{I}, z_{I}\right)$ be infinite point, i.e. $x_{I}+y_{I}+z_{I}=0$. The points $I_{A}\left(\frac{1}{2},-\frac{y_{I}}{2 x_{I}},-\frac{z_{I}}{2 x_{I}}\right), I_{B}\left(-\frac{x_{I}}{2 y_{I}}, \frac{1}{2},-\frac{z_{I}}{2 y_{I}}\right)$ and $I_{C}\left(-\frac{x_{I}}{2 z_{I}},-\frac{y_{I}}{2 z_{I}}, \frac{1}{2}\right)$ [5] together with the point $I$ form a harmonic four with respect to $\triangle A B C$. These points are finite and they lie on the lines $M_{b} M_{c}, M_{c} M_{a}, M_{a} M_{b}$. As stated in [5], the points from these lines could not be centers of conic sections, inscribed in 
$\triangle A B C$. Consequently, the point $I$ generates only one conic section, inscribed in $\triangle A B C$.

We consider the parabola $k(I)$ as a conic section with infinite center $I$. There exists exactly one parabola $k(I)$, which has an infinite center $I$ and is tangent to the lines $B C, C A$ and $A B$. The equation of the parabola $k(I)$ is $x_{I}^{2} x^{2}+y_{I}^{2} y^{2}+z_{I}^{2} z^{2}-2 y_{I} z_{I} y z-2 z_{I} x_{I} z x-2 x_{I} y_{I} x y=0$, which together with the equation of the Euler parabola $\Omega(I)$ results in a unique common point for the two curves. It has the following coordinates:

$$
F\left(-\frac{\left(y_{I}-z_{I}\right)^{2}}{9 y_{I} z_{I}},-\frac{\left(z_{I}-x_{I}\right)^{2}}{9 z_{I} x_{I}},-\frac{\left(x_{I}-y_{I}\right)^{2}}{9 x_{I} y_{I}}\right) .
$$

Thus, we establish

Theorem 2. If $I$ is an arbitrary infinite point from the plane of $\triangle A B C$, then the generated by this point inscribed parabola and Euler curve $\Omega(I)$ of $\triangle A B C$ are tangent (Fig. 6).

The case with the parabola looks like the case with hyperbola regarding the common infinite point of the inscribed parabola and the Euler curve (parabola). In such a way it turns out, that the two parabolas have two tangent points - one finite and one infinite.

The above reasoning shows, that in each concrete case all curves under consideration are of the same type.

Some interesting properties of examined constructions are contain in [6] and [7].

\section{Conclusions}

The challenge to find a generalization of a geometric theorem is connected with a deep understanding of the considered figure properties. A necessary step is to clarify the relation among the elements of a given configuration, thus extracting the properties which could be changed. How to perform the change? Which elements and properties should be modified in order to change the corresponding theorem itself? The GSP program turns out to be useful instrument in the process of answering these questions. The theorems included in the paper are mostly from the triangle geometry and are connected with different classes of circles, lines and points in the plane of that triangle. After 
the analysis of the corresponding relations the circles, the lines and the points are replaced by suitable conics, lines and points thus keeping the validity of the theorems in the new situation. A deep knowledge of conic properties and constructive skills are necessary for the purpose. The program GSP is applied for fast elimination of various conjectures which turn out to be false, but also for the creation of convincing configurations leading to the formulation of the desired assertions. The assertions themselves should be considered as true only in case they are strictly proven. Generalizations are obtained in many cases but reasons are found very often to reject some.

The established generalizations propose a new view on well-known geometric theorems and expose deeper sense of the participating figures. They give possibilities to overcome the limits of previous perceptions. Thus, a gradual deepening of the understanding concerning projective properties of conics is realized. Experience is obtained to discover certain theorems, which helps further investigations making them easier.

\section{References}

[1] Г. Паскалев, И. Чобанов. Забележителни точки в триъгълника. Народна просвета, София, 1985.

[2] И. Шарыгин. Задачи по геометрии. Планиметрия. Москва, Наука, 1986.

[3] А. Матеев. Проективна геометрия. София, Наука и изкуство, 1977.

[4] Г. Паскалев. Работата в кръжока по математика. Част II. София, Народна просвета, 1985.

[5] В. Ненков. Обобщение на теоремата на Фойербах. Математика и информатика, 2, (2008), 35 - 42.

[6] В. Ненков. Няколко свойства на Фойербаховата конфигурация. Математика и информатика, 5, (2010), 42 - 61.

[7] С. Гроздев, В. Ненков, Д. Деков. Няколко хомотетично породени свойства на фойербаховите конфигурации. Математика и информатика, 1, (2014), 68 - 72.

[8] S. Grozdev, For High Achievements in Mathematics. The Bulgarian Experience (Theory and Practice). ADE, Sofia, 2007 (ISBN 978-95492139-1-1), 295 pages.

Technical college, Lovech, Bulgaria

E-mail address: vnenkov@mail.bg 